\title{
ANÁLISE DOCUMENTAL E DE CONSISTÊNCIA TÉCNICA DE PLANOS DE MANEJO FLORESTAL EM ÁREAS DE FORMAÇÕES FLORESTAIS, NO ESTADO DE MINAS GERAIS ${ }^{1}$
}

Danilo José da Silva Coelho², Agostinho Lopes da Silva³, Carlos Pedro Boechat Soares ${ }^{3}$, Márcio Leles Romarco de Oliveira ${ }^{4}$ e Joberto Veloso de Freitas ${ }^{5}$

\begin{abstract}
RESUMO - Neste estudo foram utilizados dados de 42 planos de manejo florestal (PMF), protocolados no Instituto Estadual de Florestas no período de 1992 a 1998, com o objetivo de realizar a análise documental através de indicadores e verificadores de sustentabilidade de fácil aplicação, pertencentes à estrutura básica de um plano de manejo, conforme normas legais vigentes no Estado de Minas Gerais. Portanto, inicialmente fez-se a padronização de procedimentos referentes a todas as etapas de um PMF, tendo como base as exigências legais do Estado, de onde se originou a lista de checagem (check list) e, conseqüentemente, os indicadores e verificadores utilizados na análise documental dos PMF. Constatou-se que a ausência de um roteiro básico para elaboração dos PMF compromete a análise documental prévia para protocolo institucional e que houve deficiência institucional na análise documental e técnica dos planos de manejo. Os itens constantes dos PMF que apresentaram menor porcentagem de atendimento foram os que exigiram mais conhecimentos técnicos (análise de impacto ambiental, sistema silvicultural e bibliografia), enquanto os descritivos foram os que apresentaram maior porcentagem de atendimento e os que exigiram menos conhecimentos técnicos (objetivos e justificativas, informações gerais e discriminação de áreas da propriedade). Os indicadores e verificadores mostraram-se eficientes e de fácil aplicação nas análises documental e técnica dos planos de manejo estudados. A análise prévia documental, fundamentada em um roteiro básico elaborado neste estudo, reprovou todos os planos de manejo. É necessário padronizar e informatizar os procedimentos de análises documental e técnica para aprovação dos planos de manejo. O nível de exploração florestal, em área basal, de todos os planos de manejo analisados ultrapassou o limite máximo de intervenção permitido por lei.
\end{abstract}

Palavras-chave: Análise documental, plano de manejo florestal e florestas estacionais semideciduais.

\section{DOCUMENTAL ANALYSIS OF FOREST MANAGEMENT PLANS IN FOREST FORMATION AREAS IN MINAS GERAIS}

\begin{abstract}
This study used data from 42 forest management plans (FMP), registered at the IEF (Forest State Institute), from 1992 to 1998, aiming to perform a documental analysis employing easy to use sustainability indicators and verifiers, pertaining to the basic structure of a management plan, according to the state of Minas Gerais legal rules. Thus, all the FMP-related procedures were standardized, based on the state legal requirements, from which the checklist originated as well as the indicators and verifiers used in the FMP documental analysis. It was verified that the absence of a basic guide for the elaboration of the FMPs hinders the previous documental analyses of the institutional registration. Institutional deficiencies were detected in the documental and technical analyses of the management plans. The FMP items presenting the lowest
\end{abstract}

\footnotetext{
${ }^{1}$ Recebido em 26.04.2006 e aceito para publicação em 18.12.2007.

${ }^{2}$ Instituto Estadual de Florestas - IEF, Viçosa-MG. E-mail :<djcoelho@ufv.br>.

${ }^{3}$ Departamento de Engenharia Florestal da UFV. E-mail : <alsouza@ufv.br>.

${ }^{3}$ Departamento de Engenharia Florestal da Universidade Federal dos Vales do Jequitinhonha e Mucuri, UFVJM, Diamantina-MG. E-mail : <alsouza@ufv.br>.

${ }^{5}$ Departamento de Ciências Florestais da Universidade Federal do Amazonas (UFAM), Manaus-AM. E-mail : <joberto.freitas@florestal.gov.br>.
} 
execution percentage required greater technical knowledge (environmental impact, forestry system and references). The FMP items presenting the greatest execution percentage were descriptive and required less technical knowledge (objectives and justifications, general information and property area delimitation). The indicators and verifiers used were efficient and easy to use in the documental and technical analyses of the management plans studied. The previous documental analysis supported by a basic guide, carried out in this study disapproved all the management plans. It is necessary to standardize and compute the procedures of the documental and technical analyses for management plan approval. Forest exploitation level, in basal area, of all the management plans analyzed surpassed the maximum limit of intervention allowed by law.

Keywords: Documental analysis, forest management plan and semi-deciduous seasonal forests.

\section{INTRODUÇÃo}

A interferência humana no ambiente tem contribuído para produzir grandes mudanças, alterando, na maioria das vezes de forma negativa, os ecossistemas e afetando comunidades associadas com perda da qualidade de vida, principalmente a do homem.

Essa interferência tem causado muitos problemas aos ecossistemas florestais, principalmente com relação à qualidade de vida do Planeta. A modificação climática e o avanço das áreas degradadas são alguns dos exemplos mais conhecidos (COELHO e SOUZA, 2002).

Percebe-se, pois, que há aumento na demanda das matérias-primas florestais e também da manutenção de um mínimo de cobertura florestal nativa nas propriedades rurais, com o objetivo de proteger e conservar a biodiversidade, apontando para a necessidade de melhor organização da forma e das técnicas de utilização das florestas nativas (COELHO e SOUZA, 2005). O plano de manejo florestal é, no mínimo, uma alternativa ao desmatamento e, sobretudo, a forma de gestão das florestas para garantia da sustentabilidade.

A utilização dos recursos florestais, por meio do manejo florestal, é objeto de normatização em diversos diplomas legais. Em Minas Gerais, foi regulamentado, através da Lei Estadual no 10.561, de 27 de dezembro de 1991, com alterações introduzidas pela Lei 11.337, de 27 de dezembro de 1993, e pelo Decreto de regulamentação no 33.944, de 18 de setembro de 1992 .

Um dos primeiros diagnósticos de planos de manejo foi realizado no Estado do Pará, na microrregião de Paragominas, através do Centro de Pesquisa Agroflorestal da Amazônia Oriental (CPATU, 1996). O quadro geral revelado pelo diagnóstico foi preocupante, pois os projetos avaliados não estavam seguindo a legislação vigente e as recomendações da International Timber
Organization - ITTO (ITTO, 1990). Do ponto de vista técnico, o diagnóstico concluiu que não foi realizado manejo florestal de fato, existindo apenas atividades extrativistas de madeiras.

No Estado de Rondônia, identificou-se, através de diagnóstico, que o objetivo dos planos de manejo florestal era apenas burocrático, para atender às solicitações do Instituto Brasileiro do Meio Ambiente e dos Recursos Naturais Renováveis - IBAMA, de forma a conseguir autorizações e guias para explorar e transportar toras de madeira. Constatou-se, em todos os planos de manejo florestal diagnosticados, que não existe análise de viabilidade econômica de execução do projeto, não prevendo o custo da matéria-prima e os futuros resultados econômicos do empreendimento como um todo (CPATU, 1996).

Com relação a Minas Gerais, até o momento não se sabe a real situação dos planos de manejo florestal autorizados e em execução no Estado, sendo de extrema importância a análise documental e a avaliação da qualidade técnica desses planos, tendo em vista o conceito de sustentabilidade. A avaliação documental dos planos de manejo florestal teve como referência a legislação federal e estadual vigente no período de 1992 a 1998 e relativa ao manejo florestal e ás recomendações propostas, em sua maioria, por organizações como a ITTO (ITTO, 1992, 1998), Forest Stewardhip Council - FSC (FSC, 1998) e Center For International Forestry Research-CIFOR (CIFOR, 1996), na avaliação de planos de manejo de florestas tropicais.

Diante dessa constatação, o objetivo geral deste trabalho foi fazer a análise documental de 42 planos de manejo florestal autorizados e em execução na Zona da Mata do Estado de Minas Gerais, em áreas de Florestas Estacionais Semideciduais, utilizando-se verificadores de fácil aplicação. 
Os objetivos específicos foram:

a) Elaborar e descrever as etapas de um fluxograma de análise documental prévia e a análise de consistência técnica e vistoria de campo de um plano de manejo florestal.

b) Elaborar e descrever as etapas de um fluxograma de tramitação de um plano de manejo florestal no IEF.

c) Definir a estrutura geral de um plano de manejo florestal com base na legislação vigente na época.

d) Elaborar check list com base na estrutura geral de um plano de manejo florestal.

e) Criar verificadores de fácil aplicação na análise documental dos planos de manejo florestal protocolados no IEF. f) Avaliar, por meio de verificadores, o conteúdo dos planos de manejo florestal protocolados no IEF, no período de 1992 a 1998.

g) Estudar e definir o fluxograma das etapas de tramitação dos PMF no IEF.

\section{MATERIAL E MÉTODOS}

\section{1. Área de estudo}

\subsubsection{Caracterização da área}

A área de estudo é composta pelos Municípios de Guaraciaba, Porto Firme, Rio Espera, Teixeiras, Lamim, Senhora de Oliveira, Pedra Dourada, Matias Barbosa, Brás Pires e Piranga, localizados na mesorregião Zona da Mata, no sudeste de Minas Gerais, entre as coordenadas geográficas $19^{\circ} 45^{\prime}$ e $22^{\circ} 15^{\prime}$ latitude sul e $41^{\circ} 15^{\prime}$ e $44^{\circ} 15^{\prime}$ longitude oeste (Figura 1 ).

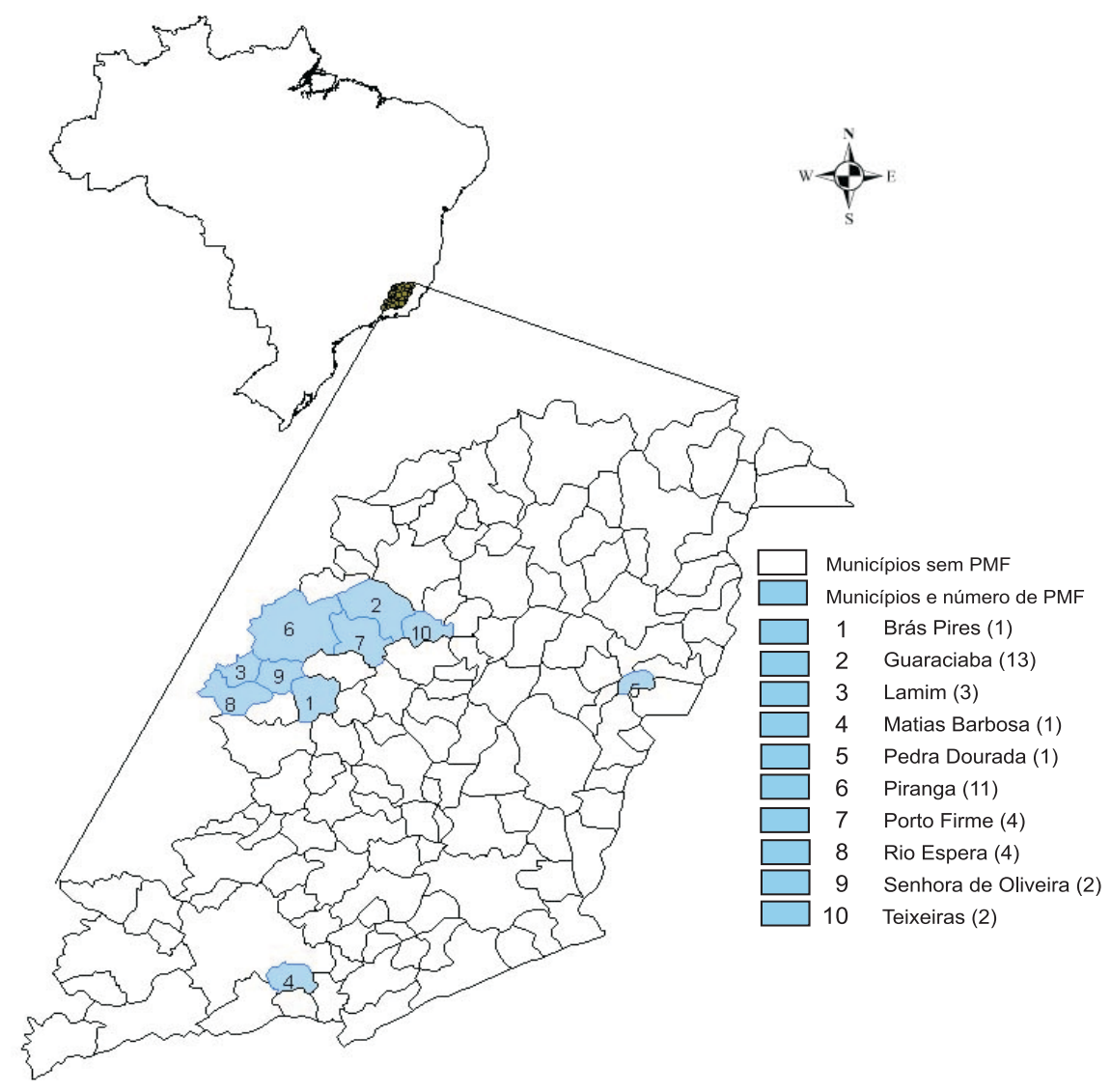

Figura 1 - Municípios onde estão localizados os planos de manejo florestal em áreas de Florestas Estacionais Semideciduais (Zona da Mata de Minas Gerais).

Figure 1 - Municipalities where the forest management plans are located in seasonal semi-decidual forests (Zona da Mata de Minas Gerais) 
A região apresenta relevo plano (7\%), ondulado (21\%) e montanhoso (72\%), com encostas de perfil convexo-côncavo embutido em vales de fundo chato, formados por terraços e leitos maiores, onde ocorrem cursos d’água. A temperatura média anual oscila em torno de $18,5^{\circ} \mathrm{C}$, e o índice médio pluviométrico anual é de aproximadamente 1.380 mm (ALMG, 2005).

A formação florestal remanescente predominante na região é a Floresta Estacional Semidecidual Submontana (situada na faixa altimétrica que varia de 100 a 600 m), nos estádios secundários de regeneração, sujeita a um ritimo estacional, com 20 a 50\% do conjunto de árvores caducifólias na época da estação seca (VELOSO et al., 1992).

\subsection{Metodologia}

Após a análise da estrutura funcional e os procedimentos administrativos do IEF, foi definido e descrito um fluxograma das etapas de tramitação de um plano de manejo florestal na instituição.

Com base na Portaria IEF-054, de 25 de agosto de 1997, que dispunha sobre as normas para elaboração de planos e manejo florestal em Minas Gerais, definiuse o formato da estrutura geral de um plano de manejo florestal, e com base nessa estrutura elaborou-se um check list para facilitar a análise documental prévia a ser feita antes do protocolo.

Através da análise do conteúdo da estrutura geral de um plano de manejo e de check list, foram criados os verificadores para análise documental de cada plano protocolado no IEF, para comprovação de sua consistência documental, conforme as exigências legais vigentes na época.

A análise documental dos 42 planos de manejo florestal protocolados no IEF, no período de 1992 a 1998, em áreas de Florestas Estacionais Semideciduais na Zona da Mata de Minas Gerais, seguiu critérios semelhantes àqueles adotados pela Receita Federal por ocasião da análise e aceitação da declaração de rendimentos de pessoa física (DRPF). O documento, isto é, o plano de manejo florestal (PMF), tem que atender a todos os itens constantes do roteiro básico fixado pelo IEF-MG, fundamentado na legislação vigente e referente ao manejo florestal. Os planos de manejo florestal aceitos para protocolo deverão atender aos 11 itens e respectivos 91 subitens. Cada item recebeu nota correspondente ao número de subitens que o acompanha e que foram atendidos. Os planos que não atenderam a todas as exigências foram considerados reprovados ,e os itens e subitens que não foram atendidos como exigência de protocolo foram apontados em análise, utilizando-se o check list. Portanto, a análise prévia, através do órgão ambiental (IEF), poderá emitir os seguintes pareceres: aprovado (podendo ser protocolado) e reprovado (devolvido ao elaborador para correções).

\section{RESULTADOS E DISCUSSÃO}

\subsection{Fluxograma de análise documental prévia de análise de consistência técnica e vistoria de campo deumPMF no IEF-MG}

A análise documental prévia seguirá critérios semelhantes àqueles adotados pela Receita Federal, por ocasião da análise e aceitação da declaração de rendimentos de pessoa física (DRPF). O documento, isto é, o plano de manejo florestal (PMF), tem que atender a todos os itens constantes do roteiro básico fixado pelo IEF-MG, fundamentado na legislação vigente e referente ao manejo florestal. Os planos de manejo florestal aceitos para protocolo deverão atender aos 11 itens e respectivos 91 subitens. Cada item recebeu a nota correspondente ao número de subitens que o acompanham e que foram atendidos. Os planos que não atenderam a todas as exigências foram considerados reprovados, e os itens e subitens que não foram atendidos como exigência de protocolo foram apontados em análise, utilizando-se o check list. Portanto, a análise prévia, através do órgão ambiental (IEF), poderá emitir os seguintes pareceres: aprovado (podendo ser protocolado) e reprovado (devolvido ao elaborador para correções).

Sumário da análise documental prévia dos planos de manejo florestal para protocolo:

1. Verificar se o documento (PMF) possui todos os itens e subitens do formato exigido pelo órgão ambiental, utilizando-se check list.

2. Emitir parecer jurídico com relação à idoneidade da documentação apresentada.

3. Apresentar parecer de aprovação ou reprovação para o protocolo.

4. No caso de reprovação, informar, em parecer, os itens e subitens que deverão ser incluídos no documento para aceitação do protocolo. 
5. Só poderão ser protocolados os PMFs que apresentarem todos os itens e subitens exigidos legalmente na formatação do documento.

A análise de consistência técnica e vistoria de campo consiste na verificação da consistência dos dados constantes no documento e da verdade de campo, por meio de vistoria técnica acompanhada de amostragem. A análise técnica possibilitará emitir, através do órgão ambiental, os seguintes pareceres: aprovado, aprovado condicionalmente e reprovado. O documento aprovado será encaminhado para o escritório local, para o técnico emitir a autorização de manejo. O documento que for aprovado, porém condicionado a adequações, será devolvido ao elaborador para proceder às correções exigidas pela análise técnica, que, após as devidas correções, submete o documento ao setor de protocolo, seguindo novamente a etapa 4 do fluxograma (Figura 2). O plano de manejo reprovado na análise técnica será arquivado na regional, e o proprietário será comunicado oficialmente do parecer, com as justificativas.

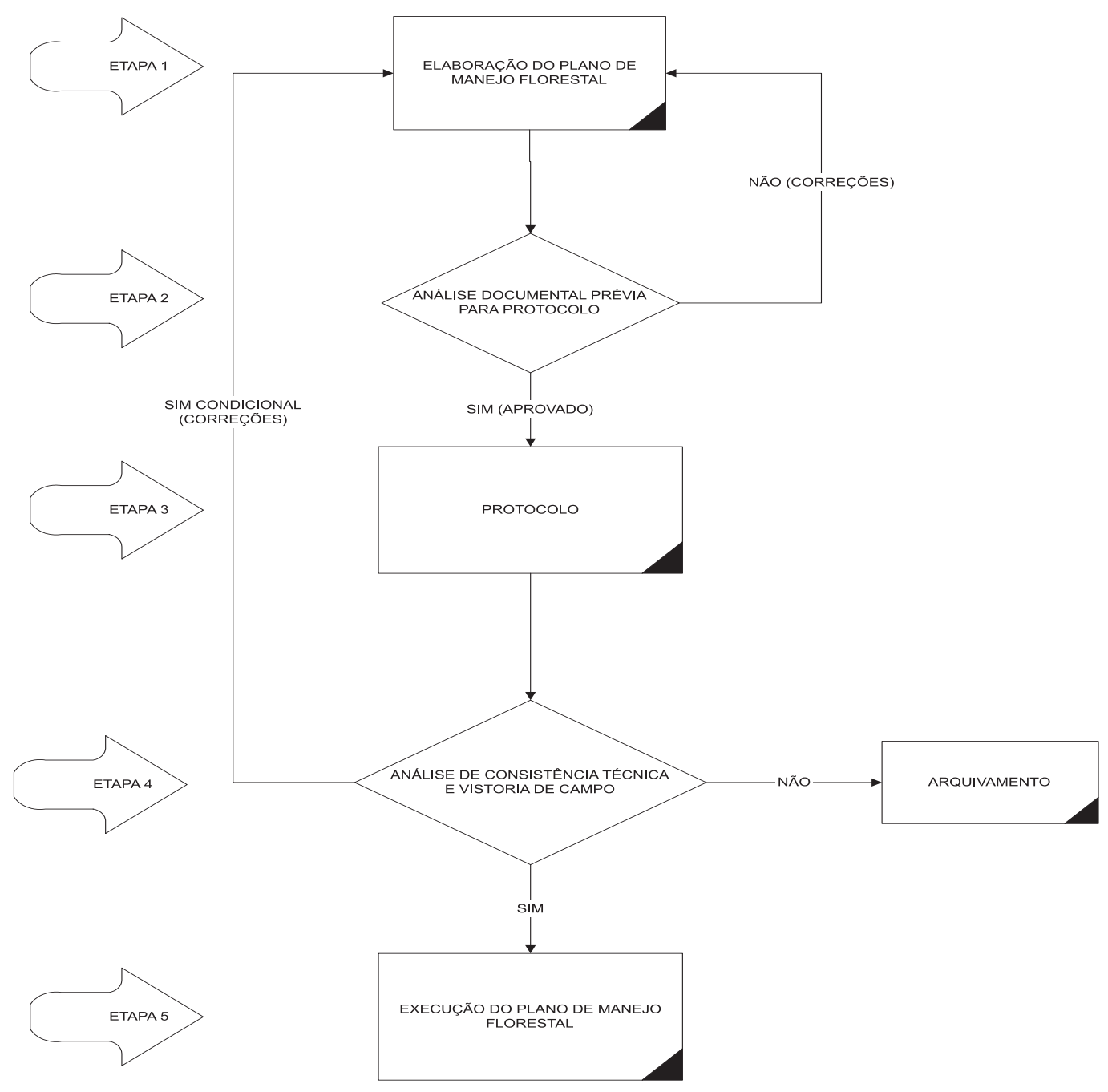

Figura 2 - Fluxograma das etapas de análise documental prévia e análise de consistência técnica e vistoria de campo de um plano de manejo florestal, no IEF-MG.

Figure 2 - Flowgrams of the stages of the previous documental analysis and technical consistency and field inspection analysis of a forest management plan 
Sumário para análise de consistência técnica e vistoria de campo:

1. Verificar o conteúdo e a consistência dos itens: inventário florestal, sistema silvicultural, níveis de intervenção e sistema de exploração.

2. Verificar, em campo, o zoneamento da propriedade, comparando as áreas com as apresentadas no mapa, com especial atenção para as áreas de reserva legal, preservação permanente e áreas passíveis de manejo.

3. Verificar a verdade de campo referente aos dados apresentados no inventário florestal, mediante a remedição de uma amostra das parcelas identificadas e delimitadas na área passível de manejo, selecionadas aleatoriamente.

4. Apresentar parecer de aprovação, aprovação condicional ou reprovação, mediante parecer técnico justificativo e informativo. No caso de aprovação condicional, informar, no parecer, os itens e subitens a serem incluídos no plano ou corrigidos, quando for o caso.

\subsection{Fluxograma das etapas de elaboração, execução e tramitação de um PMF, no IEF-MG}

Como resultado da elaboração e descrição do fluxograma (Figura 1A), foram identificadas 14 etapas referentes à tramitação dos planos de manejo florestal no IEF-MG. As etapas foram descritas com clareza e objetividade, para facilitar uma visão geral e, ao mesmo tempo detalhada de todas as etapas do processo, permitindo, quando necessário, avaliar o tempo, gasto em cada uma e interferir onde existam impedimentos ou dificuldades que, por qualquer motivo, estejam influenciando a sua normal tramitação (Quadro1A).

Todas as etapas do fluxograma são importantes e imprescindíveis e têm seqüência e ordem a serem seguidas, mas a vistoria prévia é uma etapa extremamente importante e definidora de todas as outras etapas do processo, podendo, inclusive, evitar gastos e perda de tempo desnecessário ao produtor e aos técnicos dos órgãos ambientais. É nessa etapa que é feito o pré-zoneamento da propriedade, conforme as normas legais vigentes, definindo as áreas de reserva legal, as de preservação permanente e as passíveis de manejo florestal.

\subsection{Caracterização dos planos de manejo florestal protocolados no IEF, no período de 1992 a 1998}

Os dados fornecidos pelo CPAMF-IEF (Centro de Pesquisa em Manejo - IEF) com relação aos planos de manejo protocolados no IEF, no período de 1992 a 1998, possibilitaram uma definição preliminar dos principais planos a serem avaliados por município e por estrato de área a ser manejada (Quadro1).

Do total de 42 planos de manejo florestal protocolados no período, 29 (69,04\%) possuem área de manejo menor que 10 ha, seis $(14,28 \%)$ possuem áreas entre 10 e 20 ha, três $(7,10 \%)$ entre 20 e 50 ha e dois $(4,7 \%)$ acima de 50 ha.

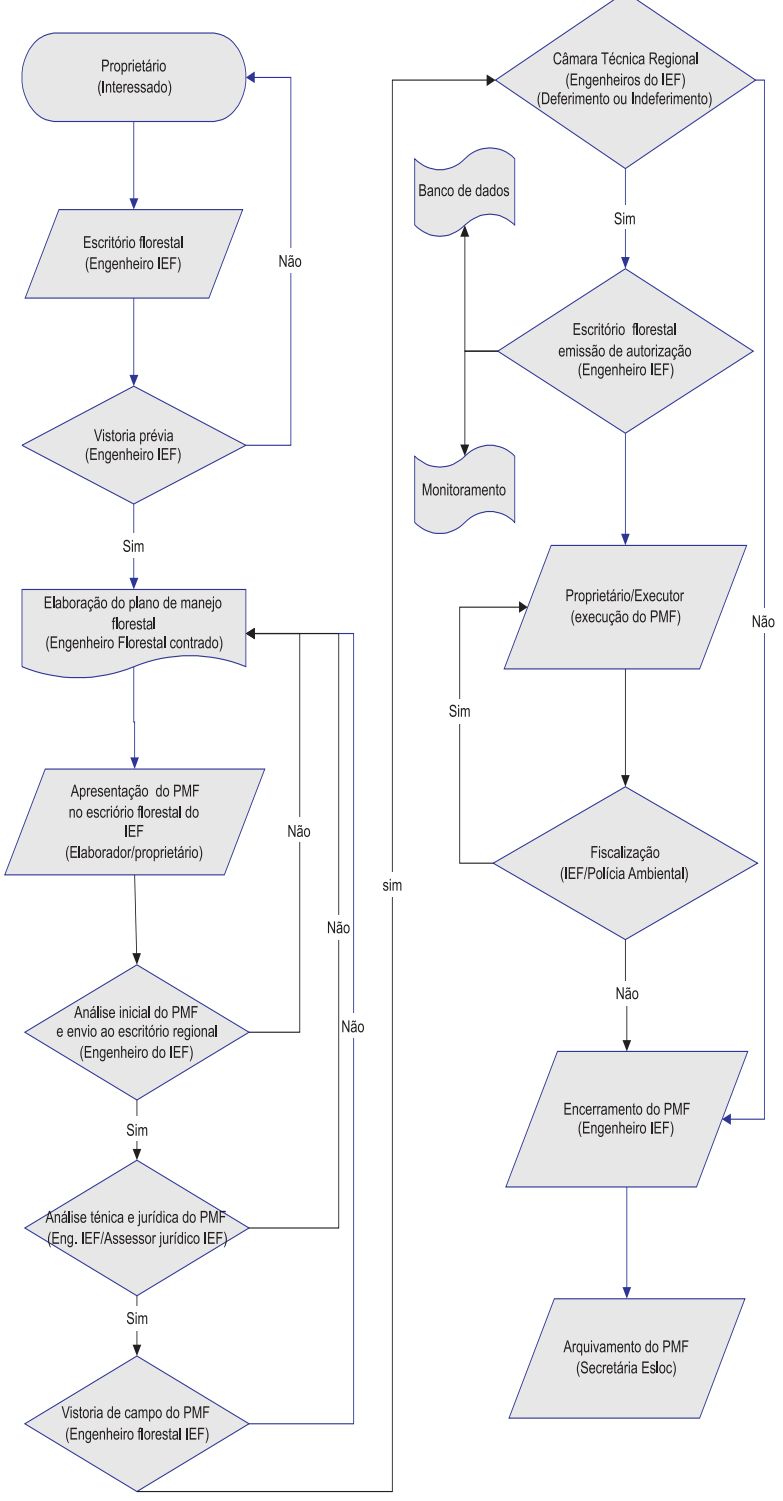

Figura 1A - Fluxograma das etapas de elaboração, execução e tramitação de um PMF no IEF-MG.

Figure 1A - Flowgram of elaboration, implementation and organization stages of a PMF at the IEF-MG 
Tabela 1 - Número de PMF por classe de área e por município, em áreas de Florestas Estacionais Semideciduais na Zona da Mata do Estado de Minas Gerais

Table 1 - Number of PMFs per area class and municipality in seasonal semidecidual forests in Zona da Mata$M G$

\begin{tabular}{|c|c|c|c|c|c|c|}
\hline \multirow[t]{2}{*}{ Município } & \multicolumn{6}{|c|}{ Área (ha) } \\
\hline & $0-10$ & $10-20$ & $20-50$ & $50-100$ & $>100$ & № de PMF \\
\hline Guaraciaba & 12 & 1 & 1 & & & 14 \\
\hline Lamim & & 2 & 1 & & & 3 \\
\hline Matias Barbosa & & & & & 1 & 1 \\
\hline Pedra Dourada & & & 1 & & & 1 \\
\hline Piranga & 9 & 2 & & & & 11 \\
\hline Porto Firme & 2 & 2 & & & & 4 \\
\hline Rio Espera & 3 & & & 1 & & 4 \\
\hline Senhora de Oliveira & 2 & & & & & 2 \\
\hline Teixeiras & 1 & 1 & & & & 2 \\
\hline Total & 29 & 08 & 03 & 1 & 1 & 42 \\
\hline$\%$ & 69,05 & 19,04 & 7,14 & 2,38 & 2,38 & 100,00 \\
\hline
\end{tabular}

Os municípios (Quadro 1) que apresentaram o maior número de planos de manejo foram Guaraciaba (33,3\%), Piranga (26,2\%), Porto Firme e Rio Espera, com 9,8\% cada. Esse fato é explicado pelo índice de cobertura florestal nativa nesses municípios, conforme verificado por IEF (1994), em que o Município de Guaraciaba apresentou 33,66\% de sua área total com cobertura florestal natural, seguido de Porto Firme, com 32,35\%; Piranga, com 30,94\%; Pedra Dourada, com 30,17\%; Matias Barbosa, com 29,31\%; Teixeiras, com 24,31\%; Lamim, 24,26\%; Senhora de Oliveira, com 20,69\%; e Rio Espera, com 19,35\%. Apenas o Município de Rio Espera apresentou área de cobertura florestal inferior a $20 \%$.

Nos termos do Código Florestal em vigor (Lei 4.771/ 1965), alterado pela Medida Provisória no 2.166-67, de 24 de agosto de 2001, a reserva legal é: área localizada no interior de uma propriedade ou posse rural, excetuada a de preservação permanente, necessária ao uso sustentável dos recursos naturais, à conservação e reabilitação dos processos ecológicos, à conservação da biodiversidade e ao abrigo e proteção de fauna e flora nativas.

A legislação florestal, então, destinou um espaço físico no interior das propriedades para cumprir os objetivos nela previstos e para que a propriedade efetivamente cumpra sua função socioambiental, conforme previsto na Constituição (FELIPE, 2005). Em termos de área física, nas propriedades situadas nas Regiões Sul e Sudeste do Brasil esse espaço deve ser no mínimo de $20 \%$ da propriedade, não podendo ser suprimida, mas apenas utilizada sob regime de manejo florestal sustentável, segundo normas e padrões técnicos estabelecidos em regulamentos. Dessa forma, em princípio a área de reserva legal é uma limitação administrativa, em que o proprietário possuidor somente pode operar se seguir determinadas regras (FELIPE, 2005).

Esse princípio, aplicado em nível territorial dos municípios estudados (Quadro 2), possibilitou a visão da porcentagem que existe de cobertura florestal, tendo como referência o limite mínimo de 20\%. Apenas o Município de Rio Espera possui cobertura florestal abaixo desse mínimo, não se considerando as áreas de preservação permanente e outras áreas especialmente protegidas (IEF, 1998).

\subsection{Análise documental e classificação dos planos de manejo florestal (PMF), com base em indicadores e verificadores}

Todos os planos de manejo florestal foram analisados com base nos 11 verificadores extraídos da estrutura geral de um plano e classificados de acordo com o peso de cada verificador, com a nota máxima obtida por cada um.

Os PMF que obtiveram as maiores notas foram: $\mathrm{PMF}_{15}$ (71), $\mathrm{PMF}_{41}$ (70) e $\mathrm{PMF}_{40}$ (69). Os PMF que tiveram as menores notas foram: $\mathrm{PMF}_{31}(37), \mathrm{PMF}_{13}$ (44) e $\mathrm{PMF}_{11}$ (46).

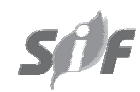

R. Árvore, Viçosa-MG, v.32, n.1, p.69-80, 2008 
Tabela 2 - Cobertura florestal nativa, por município, na Zona da Mata de Minas Gerais, em 1998 (IEF, 1998) Table 2 - Native forest cover per municipality in Zona da Mata -MG, in 1998 (IEF, 1980)

\begin{tabular}{lcccc}
\hline Município & Área Total $(\mathrm{ha})$ & Altitude $(\mathrm{m})$ & Floresta Nativa (ha) & Porcentagem (\%) \\
\hline Guaraciaba & $34.866,00$ & 580 & $11.737,25$ & 33,66 \\
Porto Firme & $28.521,00$ & 600 & $9.226,26$ & 32,35 \\
Piranga & $65.915,00$ & 600 & $20.395,71$ & 30,94 \\
Pedra Dourada & $7.012,00$ & 650 & $2.115,72$ & 30,17 \\
Matias Barbosa & $15.665,00$ & 476 & $4.591,24$ & 29,31 \\
Teixeiras & $16.609,00$ & 649 & $4.038,30$ & 24,31 \\
Lamim & $11.847,00$ & 760 & $2.874,15$ & 24,26 \\
Senhora de Oliveira & $17.063,00$ & 710 & $3.530,25$ & 20,69 \\
Rio Espera & $23.903,00$ & 690 & $4.626,36$ & 19,35 \\
\hline
\end{tabular}

Fonte: Base de dados da Coordenadoria de Monitoramento e Controle, CEDEF-IEF-Viçosa-MG.

Os itens com menor porcentagem de atendimento foram análise de impactos (4,8\%), sistema silvicultural e níveis de intervenção(38,1\%) ebibliografia(40,5\%). Os itens commaior porcentagem de atendimento foram: objetivos e justificativas (97,6\%), informações gerais (96,2\%) e discriminação na área da propriedade (69,7\%) (Figura 4).

Observou-se que nenhum dos itens foram plenamente atendidos e que os de fundamental importância para a sua aprovação e execução correta, como inventário florestal, sistema silvicultural e níveis de intervenção e sistema de exploração, não foram apresentados, conforme exigências legais.
Esses dados indicam que há necessidade de padronização de todas as etapas do manejo e implantação de um sistema de informatização, com o objetivo de melhorar o controle e checagem de cada item, eliminando no protocolo as pendências que podem comprometer, legal e tecnicamente, os projetos apresentados. Concomitantemente com esses procedimentos, realizou o treinamento de técnicos responsáveis pela elaboração e análise, com ênfase nas etapas que apresentaram maiores índices de falhas, visando à melhor qualidade, o que, conseqüentemente, resulta na melhor execução e monitoramento.

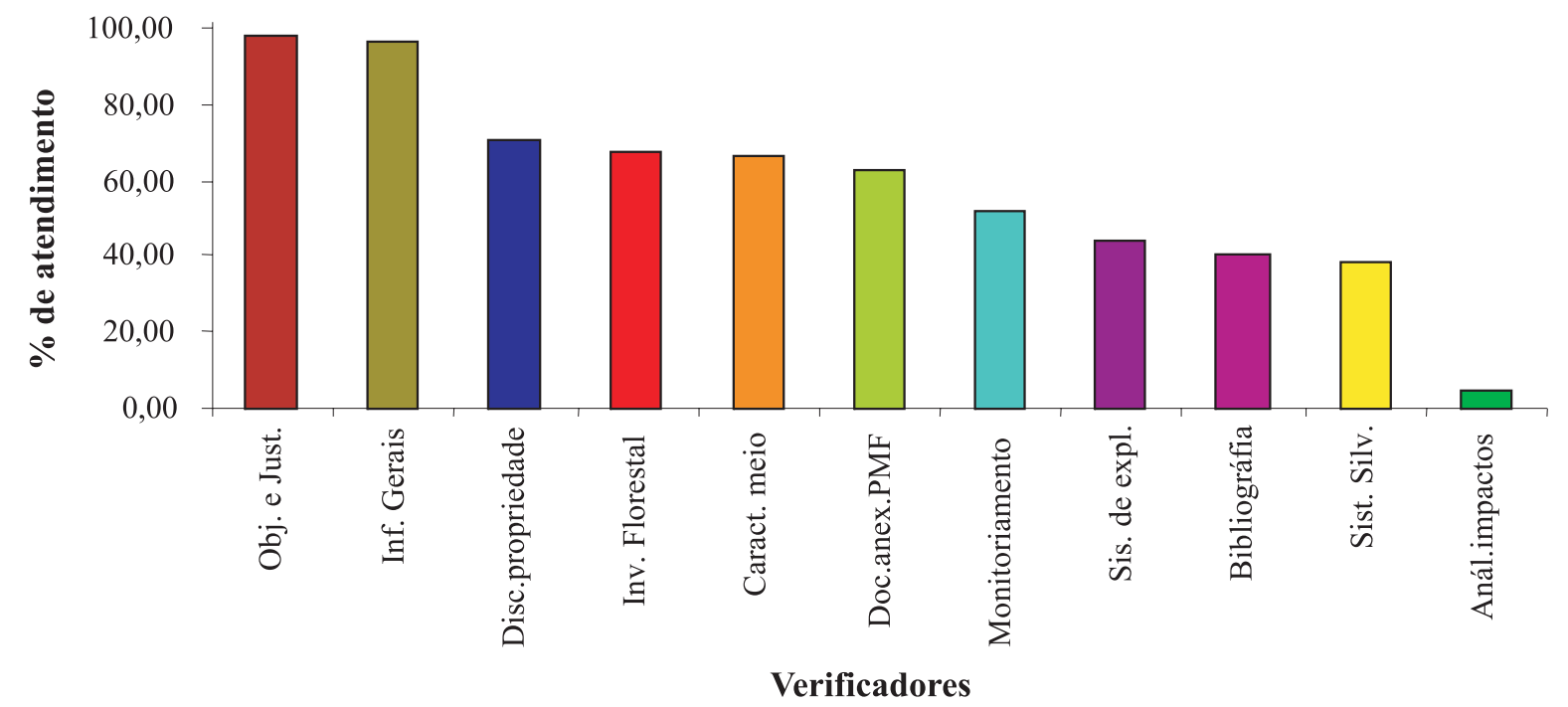

Figura 4 - Porcentagem de atendimento de cada verificador após a análise dos 42 PMF, em áreas de formações florestais, na Zona da Mata, Estado de Minas Gerais.

Figure 4 - Attendance percentage of each verifier after analyzing the $42 \mathrm{PMFs}$ in forest formation areas in Zona da Mata, $M G$.

R. Árvore, Viçosa-MG, v.32, n.1, p.69-80, 2008 
Após a análise documental, com base nos indicadores e verificadores, obteve-se o desempenho dos planos, de acordo com o atendimento dos quesitos básicos oriundos de uma estrutura geral de um plano de manejo, conforme normas legais vigentes na época. Nenhum dos 42 PMF (100,0\%) atingiu a nota mínima de 91 pontos, portanto todos foram reprovados (Figura 5). Os planos protocolados no IEF não atenderam às regulamentações técnicas legalmente exigidas (Quadro 3), no entanto foram aprovados e autorizados a realizar a exploração florestal.

\subsection{Análise de consistência técnica (nível de intervenção em área basal) dos planos de manejo florestal estudados}

Os resultados do estoque de área basal da vegetação arbórea antes e após a primeira exploração florestal, dos $\mathrm{PMF}_{28}, \mathrm{PMF}_{29}, \mathrm{PMF}_{30}$ e $\mathrm{PMF}_{16}$, encontram-se no Quadro 4.

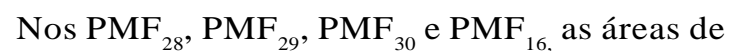
manejo florestal apresentaram, respectivamente, 14,28; 7,42; 9,99; e 16,67 m² ha-1 de área basal, e as áreas de reserva legal apresentaram 17,56; 10,16; 9,87; e 17,40 $\mathrm{m}^{2}$ ha $^{-1}$. Considerando um nível de intervenção de $60 \%$ das áreas basais originais, ou seja, antes da primeira exploração, e obtidas nos resultados dos inventários florestais dos planos (Quadro 4) sendo verificadores de obediência às leis e normas vigentes à época (IEF,
054/1997), as áreas basais remanescentes no início do processo de regeneração deveriam, respectivamente, ser de 15,64; 11,62; 11,62; 13,69; e 18,09 m² ha-1. Após a exploração, a área basal de cada plano de manejo estudado evidenciou que o estoque remanescente é de 25,6\% no $\mathrm{PMF}_{29}$, 29,2\% no $\mathrm{PMF}_{30}, 36,5 \%$ no $\mathrm{PMF}_{28}$ e 48,7\% no $\mathrm{PMF}_{16}$, em comparação com o estoque original. Diante dos resultados, pode-se inferir que essas eram áreas de florestas secundárias, que os níveis de intervenções na época da primeira exploração florestal ultrapassaram os limites legais permitidos (Figura 6).

O verificador distribuição de área basal por classe de dap apontou diferenças não-significativas $(\mathrm{P}>0,05)$ para os $\mathrm{PMF}_{28}, \mathrm{PMF}_{29}$ e $\mathrm{PMF}_{30}$ e significativa $(\mathrm{Ps} \leq 0,05)$ para o $\mathrm{PMF}_{16}$, entre as áreas de manejo florestal e áreas de reserva legal, em 2005 (Quadro 5).

Tabela 3 - Resultado da avaliação do desempenho documental dos $42 \mathrm{PMF}_{\mathrm{s}}$, em áreas de formações florestais, na Zona da Mata do Estado de Minas Gerais

Table 3 - Results of the documental performance evaluation of the 42 PMFs in forest formation areas in Zona da Mata-MG

\begin{tabular}{llcr}
\hline Pontuação & $\begin{array}{l}\text { Nível de } \\
\text { Qualidade }\end{array}$ & $\begin{array}{l}\text { Número } \\
\text { de PMF }\end{array}$ & Porcentagem \\
\hline 91 pontos & Aprovado & 0 & 00,00 \\
$<91$ pontos & Reprovado & 42 & 100,00 \\
\hline Total & & 42 & 100,00 \\
\hline
\end{tabular}

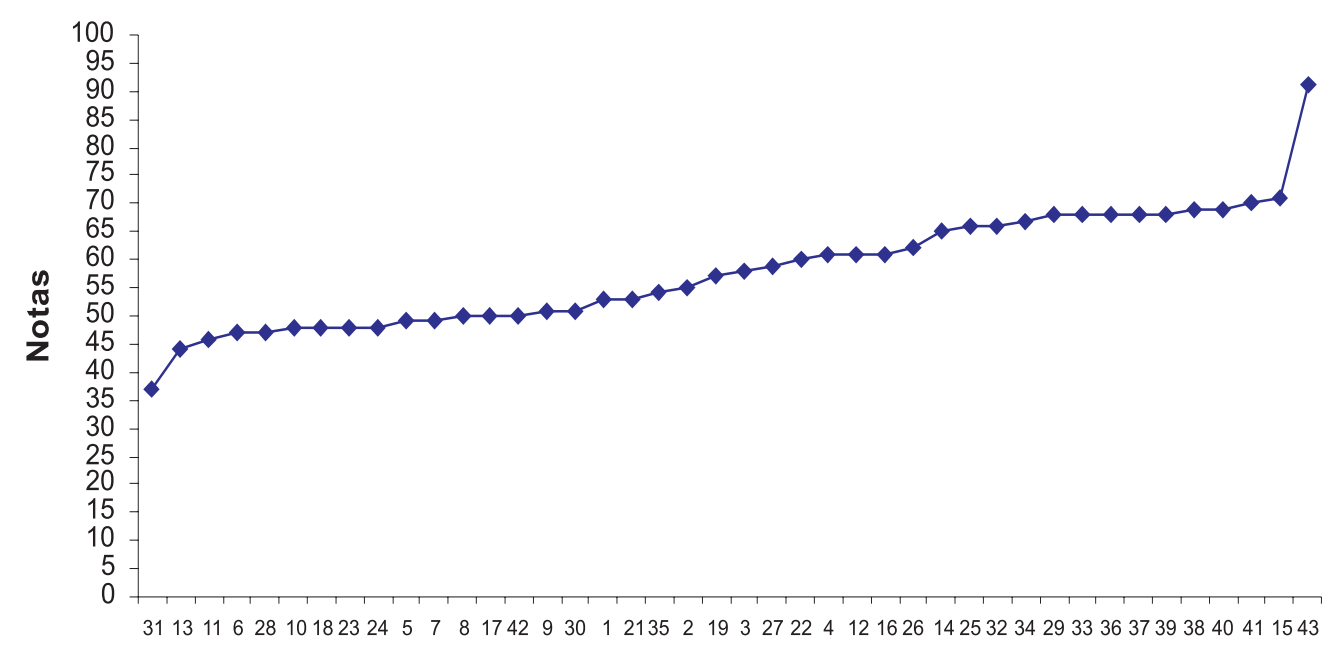

Planos de Manejo florestal

Figura 5 - Pontuação dos 42 PMF, em áreas de formações florestais, na Zona da Mata do Estado de Minas Gerais, após a análise documental utilizando verificadores.

Figure 5 - Scores of the 42 PMFs, in forest formation areas in Zona da Mata-MG,after documental analysis using verifiers. 
Tabela 4 - Estoques de área basal $\left(\mathrm{m}^{2} \mathrm{ha}^{-1}\right)$, para DAP $\geq 5,0 \mathrm{~cm}$, antes e depois da exploração florestal das áreas de manejo autorizadas nos $\mathrm{PMF}_{28}, \mathrm{PMF}_{29}, \mathrm{PMF}_{30}$ e $\mathrm{PMF}_{16}$, nos Municípios de Porto Firme e Rio Espera, Minas Gerais

Table 4 - Basal area stocks $\left(\mathrm{m}^{2} \mathrm{ha}^{-1}\right)$ for $D A P \geq 5.0 \mathrm{~cm}$, before and after forest exploration of the management areas authorized at the $P M F_{28}, P M F_{29}, P M F_{30}$ and $P M F_{16}$ in Porto Firme and Rio da Espera $-M G$

\begin{tabular}{|c|c|c|c|c|c|c|}
\hline \multirow[t]{2}{*}{ PMF } & \multirow[t]{2}{*}{ Ano } & \multicolumn{2}{|c|}{ Área Basal $\left(\mathrm{m}^{2} \mathrm{ha}^{-1}\right)$} & \multirow[t]{2}{*}{ Ano } & \multicolumn{2}{|c|}{ Área Basal $\left(\mathrm{m}^{2} \mathrm{ha}^{-1}\right)$} \\
\hline & & AMF & ARL & & AMF & ARL \\
\hline $\mathrm{PMF}_{28}$ & 1995 & $39,12^{1}$ & - & 2005 & $14,28^{2}$ & $17,56^{2}$ \\
\hline $\mathrm{PMF}_{29}$ & 1997 & $29,06^{1}$ & - & 2005 & $7,42^{2}$ & $10,16^{2}$ \\
\hline $\mathrm{PMF}_{30}$ & 1996 & $34,24^{1}$ & - & 2005 & $9,99^{2}$ & $9,87^{2}$ \\
\hline $\mathrm{PMF}_{16}$ & 1994 & $45,23^{1}$ & - & 2005 & $16,67^{2}$ & $17,40^{2}$ \\
\hline
\end{tabular}

${ }^{1}$ Dados oriundos das planilhas de inventário florestal dos PMF.

${ }^{2}$ Dados oriundos da amostragem dos PMF realizada em 2005.

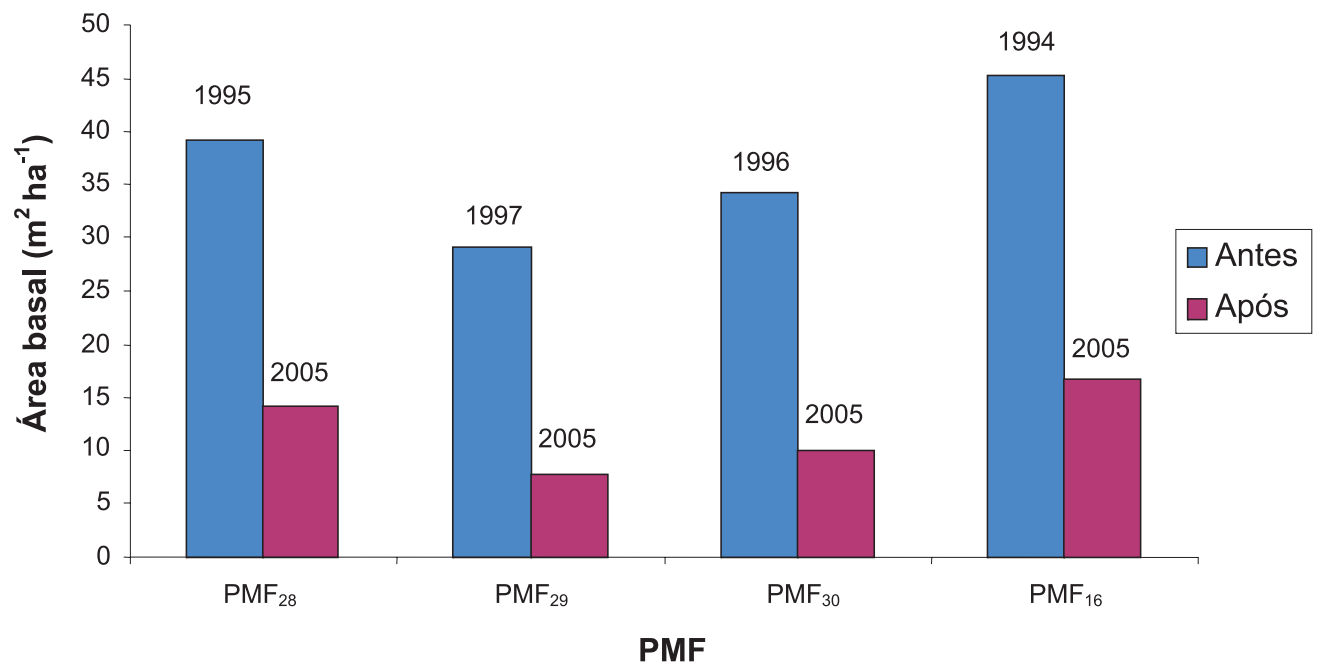

Figura 6 - Área basal antes (1994, 1995, 1996, 1997) e depois (2005) da exploração florestal das espécies arbóreas dos $\mathrm{PMF}_{28}, \mathrm{PMF}_{29}, \mathrm{PMF}_{30}$ e PMF 16 , nos Municípios de Porto Firme e Rio Espera, Minas Gerais.

Figure 6 -Basal area before $(1994,1995,1996,1997)$ and after(2005) forest exploration of the tree species of the $P M F_{28}$, $P M F_{29}, P M F_{30}$ and $P M F_{16}$ ) in Porto Firme and Rio da Espera-MG.

Tabela 5 - Resultado do teste L \& O, comparando as distribuições de área basal das espécies arbóreas por classe de diâmetro, ocorrentes em área de manejo florestal e área de reserva legal dos $\mathrm{PMF}_{28}, \mathrm{PMF}_{29}, \mathrm{PMF}_{30}$ e $\mathrm{PMF}_{16}$, nos Municípios de Porto Firme e Rio Espera, Minas Gerais

Table 5 - Result of the L\&O test comparing the basal area distributions of the tree species per diameter class, in forest management area and legal reserve area of the $P M F_{28}, P M F_{29}, P M F_{30}$ and $P M F_{16}$, in Porto Firme and Rio da Espera - MG

\begin{tabular}{llclll}
\hline PMF & Parâmetro & $\mathrm{F}\left(\mathrm{H}_{0}\right)$ & $\mathrm{t}$ & $r_{Y_{Y_{1}}}$ & Decisão \\
\hline $\mathrm{PMF}_{28}$ & Área basal $\left(\mathrm{m}^{2} \mathrm{ha}^{-1}\right)$ & $\mathrm{ns}$ & $\mathrm{ns}$ & $r_{Y_{Y_{1}}} \geq\left(1-\left.\right|_{\bar{e}} \mid\right)$ & $Y_{j}=Y_{1}$ \\
$\mathrm{PMF}_{29}$ & Área basal $\left(\mathrm{m}^{2} \mathrm{ha}^{-1}\right)$ & $\mathrm{ns}$ & $\mathrm{ns}$ & $r_{Y_{Y_{1}}} \geq(1-|\bar{e}|)$ & $Y_{j}=Y_{1}$ \\
$\mathrm{PMF}_{30}$ & Área basal $\left(\mathrm{m}^{2} \mathrm{ha}^{-1}\right)$ & $\mathrm{ns}$ & $\mathrm{ns}$ & $r_{Y_{Y_{1}}} \geq\left(1-\left.\right|_{\bar{e}} \mid\right)$ & $Y_{j}=Y_{1}$ \\
$\mathrm{PMF}_{16}$ & Área basal $\left(\mathrm{m}^{2} \mathrm{ha}^{-1}\right)$ & $*$ & $\mathrm{~ns}$ & $r_{Y_{Y_{1}}} \leq\left(1-\left.\right|_{\bar{e}} \mid\right)$ & $Y_{j} \neq Y_{1}$ \\
\hline
\end{tabular}

* Significativo a 5\% e ns - não-significativo a 5\%, F $\left(\mathrm{H}_{0}\right)=$ teste $\mathrm{F}$ de Graybill, $\mathrm{t}_{\bar{e}}=$ teste t para o erro médio, $r_{Y_{Y} Y_{1}}=$ coeficiente de correlação linear, $Y_{1}=$ área explorada e $Y_{\mathrm{j}}=$ área de reserva legal.

R. Árvore, Viçosa-MG, v.32, n.1, p.69-80, 2008 


\section{CONCLUSÕES}

Com base na análise documental e de consistência técnica de 42 planos de manejo florestal protocolados no IEF, no período de 1992 a 1998, e utilizando indicadores e verificadores, chegou-se às seguintes conclusões:

A ausência de um roteiro básico para elaboração dos PMFs compromete a análise documental prévia para protocolo institucional.

Houve deficiências institucional na análise documental e técnica nos planos de manejo estudados.

Os itens constantes dos PMFs que apresentaram menor porcentagem de atendimento foram os que exigiram mais conhecimentos técnicos (análise de impacto ambiental, sistema silvicultural e bibliografia).

Os itens constantes dos PMFs que apresentaram maior porcentagem de atendimento, e que exigiram menos conhecimentos técnicos (objetivos e justificativas, informações gerais e discriminação de áreas da propriedade) foram os descritivos.

Os indicadores e verificadores mostraram-se eficientes e de fácil aplicação nas análises documental e técnica dos planos de manejo estudados.

A análise prévia documental, fundamentada em roteiro básico elaborado neste estudo, reprovou todos os planos de manejo.

É necessário padronizar e informatizar os procedimentos de análises documental e técnica para aprovação dos planos de manejo.

O nível de exploração florestal, em área basal, de todos os planos de manejo analisados ultrapassou o limite máximo de intervenção permitido por lei.

\section{REFERÊNCIAS}

ASSEMBLÉIA LEGISLATIVA DO ESTADO DE MINAS GERAIS - ALMG caracterização dos municípios mineiros com base em informações do IGA-CETEC e IBGE. Disponível em: <www.almg.gov.br>. Acesso em: 7 dez. de 2005.
CENTER FOR INTERNATIONAL FORESTRY

RESEARCH - CIFOR. Testing criteria and indicators for the sustainable management of forests: Phase 1. Indonésia: 1996. p.2-72. (Final report)

CENTRO DE PESQUISAAGROFLORESTAL DA AMAZÔNIA ORIENTAL - CPATU.

Diagnóstico dos projetos de manejo florestal no Estado do Pará - Fase Paragominas. [s. 1.]:1996. 92p. (Relatório)

COELHO, D. J. S.; SOUZA, A. L. Modelo de gestão florestal sustentável para microrregião de Viçosa, Minas Gerais. Revista Árvore, v.26, n.2, p.145-154, 2002.

COELHO, D. J. S.; SOUZA, A. L.; OLIVEIRA, C. M. L. Levantamento da cobertura florestal natural da microrregião de Viçosa, Minas Gerais, utilizando-se imagens de Landsat 5. Revista Árvore, v.29, n.1, p.17-24, 2005.

FELIPE, J. O. A legislação ambiental e as áreas de reserva legal florestal. Disponível em: <http://www.1.jus.com.br/ doutrina $>$. Acesso em: 2 maio de 2005.

FOREST STEWARDSHIP COUNCIL - FSC. Padrões de certificação do FSC para manejo florestal em terra firme na Amazônia Brasileira. Brasília: Grupo de Trabalho do FSC no Brasil, 1998. 33p.

(Documento, 2.0)

INSTITUTO ESTADUAL DE FLORESTAS - IEF.

Cobertura vegetal e uso do solo do Estado de Minas Gerais. Viçosa, MG: Base de dados da Coordenadoria de Monitoramento e Controle, CEDEF-IEF, 1998.

INSTITUTO ESTADUAL DE FLORESTAS - IEF. Contrato BIRD/ SEPLAN/IEF-MG. Programa Prófloresta. Cobertura vegetal e uso do solo do Estado de Minas Gerais. Belo Horizonte: 1994. 1 mapa: color. 99x66. Escala: 1:1.500.000. Material cartográfico.

INTERNATIONAL TIMBER ORGANIZATION ITTO. Atualização dos critérios para a avaliação de manejo sustentado de florestas tropicais. Yokihama: ITTO, 1998. 22p. (Série Política de Desenvolvimento, 7).

R. Árvore, Viçosa-MG, v.32, n.1, p.69-80, 2008 
INTERNATIONAL TIMBER ORGANIZATION - ITTO. Criteria for the measurement of sustainable tropical forest management. Yokihama: 1992. (ITTO-Policy Development Series, 3)

INTERNATIONAL TIMBER ORGANIZATION ITTO. Guidelines for the sustainable management of natural tropical forests. Yokihama: 1990. (ITTO Technical Seires, 5).
LEITE, H. G.; OLIVEIRA, F. H. T. Statistical procedure to test the identity of analytical methods. Comm. Soil Sci. Plant Anal., v. 33, n.7/8, p.1105-1118, 2002.

VELOSO, H. P.et al. Manual técnico da vegetação brasileira. Rio de Janeiro: IBGE, 1992. 92p. (Série Manuais Técnicos em Geociências, 1). 